\title{
Bone mineral density in patients with obstructive sleep apnea syndrome
}

\author{
Sophia E. Schiza • Izolde Bouloukaki • \\ Charalampos Mermigkis
}

Received: 6 March 2012 /Revised: 6 March 2012 / Accepted: 20 March 2012 /Published online: 13 April 2012

(C) Springer-Verlag 2012

In the past two decades, obstructive sleep apnea syndrome (OSAS) has been identified as a common clinical condition with high prevalence in middle-aged adults [1]. OSAS may trigger a cascade of intermediate mechanisms that are potentially harmful to the cardiovascular and metabolic system, and include sympathetic activation activity, oxidative stress, and systemic inflammation [2]. All these intermediate mechanisms may help to explain the contribution of OSAS to several diseases, including hypertension, stroke, diabetes, and cardiac arrhythmias and the increased mortality associated with the disease [3-6]. Cardiovascular morbidity and mortality associated with sleep apnea have been extensively studied in the last decade $[4,7,8]$. Furthermore, several former and recent studies have indicated strong association between cardiovascular diseases and osteoporosis [9-13], with the above intermediate mechanisms of OSAS to have a critical role in the common pathophysiological pathway of both diseases. Therefore, this association leads us to hypothesize that OSAS may also be responsible for the development of osteoporosis.

In this issue of Sleep and Breathing, Hulya Uzkeser, and colleagues of the Ataturk University, Turkey, in their paper "Bone mineral density in patients with obstructive sleep apnea syndrome" tried to investigate the relationship between OSAS and osteoporosis by examining both the bone metabolic abnormalities and bone mineral density (BMD) in OSAS patients compared to individuals without OSAS. The authors found a significant difference between OSAS patients and the control group in relation to lumbar L1-L4

S. E. Schiza $(\bowtie) \cdot$ I. Bouloukaki $\cdot$ C. Mermigkis

Sleep Disorders Unit, Department of Thoracic Medicine,

University General Hospital,

Medical School of the University of Crete,

71110, Heraklion, Crete, Greece

e-mail: schiza@med.uoc.gr
BMD, femoral neck BMD, and lumbar L1-L4 $t$ score values, evaluating the underlying assumption of the relationship between OSAS and osteoporosis. Taking into account that there have been no previous reports about the correlation between OSAS and osteoporosis, assessed by bone mineral density, we appreciate this type of work. Furthermore, the study certainly has clinical importance as the issue of osteoporosis is very important, underestimated, and with rising prevalence. Many well-designed epidemiologic studies worldwide have demonstrated that this disease and its consequences represent a high burden on health systems due to the high costs and the resources needed for the care of patients suffering from it [14].

What can these results be attributed to? There are at least three mechanisms that may potentially affect abnormal bone metabolism in OSAS: hypoxia, increased oxidative stress, and inflammation [15]. As the authors hypothesize, the balance between osteoclasts and osteoblasts is disrupted in favor of osteoclasts in the presence of hypoxia. However, the fact that they did not find any significant differences in serum Ca, P, ALP, and urinary Dpd levels in OSAS patients compared to controls is still not clear.

In the literature, the first clinical indication of the adverse effects of OSAS on bone metabolism was by Tomiyama et al. [15]. The authors investigated the association between the severity of OSAS and the biochemical markers of bone metabolism in 50 male patients with OSAS and 15 controls, and also tested the effect of CPAP therapy. They found that severe OSAS was associated with increased bone resorption, assessed by urinary excretion of cross-linked C-terminal telopeptide of type I collagen (CTX), suggesting that the abnormal bone metabolism in OSA is characterized by "an uncoupled state" between increased bone resorption and relatively suppressed bone formation. They showed further that the urinary CTX was reversed following 3 months' CPAP 
therapy, possibly due to reduced inflammation and oxidative stress along with improvement in the arterial oxygen saturation associated with CPAP therapy. However, convincing data from large studies confirming such an association are again lacking. Moreover, as the authors explain, the urinary CTX levels showed no significant correlations with the plasma levels of any of the inflammatory cytokines or of a marker of oxidative stress after adjustment for other variables, either at the baseline or after 3 months' CPAP therapy, suggesting that the inflammation or oxidative stress associated with OSAS may affect partially but not strongly bone metabolism. To the best of our knowledge, there are no other reports about the correlation between OSAS and osteoporosis.

The strengths of this study include the use of the current gold standard diagnostic method in the diagnosis of osteoporosis, DEXA, to evaluate osteoporosis, the prospective collection of data, and the objective documentation of sleep abnormalities with attended overnight polysomnography. However, the readers should bear in mind that this study did not include any BMI-matched control subjects. Furthermore, the authors did not address other factors potentially involved in both OSAS and bone metabolic abnormalities, such as the influence of cortisol and physical activity, which may be helpful for elucidating the pathophysiological link between OSAS and osteoporosis.

Nevertheless, this study adds to emerging literature suggesting OSAS as a risk factor for osteoporosis. Ideally, these data suggest the need for well-conducted prospective studies with bone mineral density and/or fracture incidence as endpoints to determine whether or not OSAS is indeed an independent risk factor for osteoporosis and whether treating sleep apnea enables osteoporosis to be reversed, particularly in comparison with usual treatment strategies.

In conclusion, OSAS and osteoporosis are two increasingly prevalent disorders that appear to be related. The nature of this association remains to be clarified, but a number of pathophysiological pathways are potential mediating mechanisms. The study by Uzkeser and colleagues provides further rationale to add OSAS to dietary factors, medications, tobacco use, and lack of exercise as crucial factors that need to be modified to curb the osteoporosis epidemic. Further longitudinal and interventional studies are required to help confirm these observations and assess the impact of CPAP therapy on the bone mass and quality in subjects with OSAS.

\section{References}

1. Young T, Peppard PE, Gottlieb DJ (2002) Epidemiology of obstructive sleep apnoea: a population health perspective. Am J Respir Crit Care Med 165:1217-1239

2. Lévy P, Tamisier R, Minville C, Launois S, Pépin JL (2011) Sleep apnoea syndrome in 2011: current concepts and future directions. Eur Respir Rev 20:134-146

3. Gami AS, Howard DE, Olson EJ, Somers VK (2005) Day-night pattern of sudden death in obstructive sleep apnea. N Engl J Med 352:1206-1214

4. Marin JM, Carrizo SJ, Vicente E, Agusti AG (2005) Long-term cardiovascular outcomes in men with obstructive sleep apnoeahypopnoea with or without treatment with continuous positive airway pressure: an observational study. Lancet 365:1046-1053

5. Arzt M, Young T, Finn L, Skatrud JB, Bradley TD (2005) Association of sleep-disordered breathing and the occurrence of stroke. Am J Respir Crit Care Med 172:1447-1451

6. Mehra R, Benjamin EJ, Shahar E, Gottlieb DJ, Nawabit R, Kirchner HL, Sahadevan J, Redline S, Sleep Heart Health Study (2006) Association of nocturnal arrhythmias with sleep-disordered breathing: the Sleep Heart Health Study. Am J Respir Crit Care Med 173:910-916

7. McNicholas WT, Bonsignore MR, COST action B26 MC (2007) Sleep apnoea as an independent risk factor for cardiovascular disease: current evidence, basic mechanisms and research priorities. Eur Respir J 29:156-178

8. Punjabi NM, Caffo BS, Goodwin JL, Gottlieb DJ, Newman AB, O'Connor GT, Rapoport DM, Redline S, Resnick HE, Robbins JA, Shahar E, Unruh ML, Samet JM (2009) Sleep-disordered breathing and mortality: a prospective cohort study. PLoS Med 6: e1000132

9. Aronow WS (2011) Osteoporosis, osteopenia and atherosclerosis vascular disease. Arch Med Sci 7:21-26

10. Seo SK, Cho S, Kim HY, Choi Y, Choi YS, Park KH, Cho DJ, Lee BSS, Park KH, Cho DJ, Lee BS (2009) Bone mineral density, arterial stiffness, and coronary atherosclerosis in healthy postmenopausal women. Menopause 16:937-943

11. Von der Recke P, Hansen MA, Hassager C (1999) The association between low bone mass at the menopause and cardiovascular mortality. Am J Med 106:273-278

12. Vogt MT, Cauley JA, Kuller LH, Nevitt MC (1997) Bone mineral density and blood flow to the lower extremities: the study of osteoporotic fractures. J Bone Miner Res 12:283-289

13. Salari Sharif P, Abdollahi M (2011) A systematic review on the relationship between use of statins and osteoporosis. Int $\mathrm{J}$ Pharmacol 7:180-188

14. Johnell O, Kanis JA (2004) An estimate of the worldwide prevalence, mortality and disability associated with hip fracture. Osteoporos Int 15:897-902

15. Tomiyama H, Okazaki R, Inoue D, Ochiai H, Shiina K, Takata Y, Hashimoto H, Yamashina A (2008) Link between obstructive sleep apnea and increased bone resorption in men. Osteoporos Int 19:1185-1192 